\title{
A time-domain precise BEM for solving transient heat conduction problems with variable heat conductivities
}

\author{
Bo Yu, Wei-An Yao, Xiao-Wei Gao \\ State Key Laboratory of Structural Analysis for Industrial Equipment, \\ Dalian University of Technology, China
}

\begin{abstract}
In this paper, the Green's function for the Laplace equation is adopted to derive the boundary integral equation for solving transient heat conduction problems with variable heat conductivities and heat sources. As a result, domain integrals are involved in the derived integral equations. Firstly, the radial integration method is used to convert the domain integrals into equivalent boundary integrals. Then, by expanding variables at a discrete time interval, the recursive formulation of the governing equation is derived. Finally, the recursive equation is solved by the radial integration boundary element method. A self-adaptive check technique is carried out to estimate how many expansion terms are needed in a time step size. Numerical results show satisfactory performance.
\end{abstract}

Keywords: time-domain precise algorithm, radial integration method, boundary element method, self-adaptive check, variable heat conductivity, heat source.

\section{Introduction}

Transient heat conduction problems can be considered as the discretization of space and time domain. There are many methods to discretize the space domain, such as the finite element method (FEM), the boundary element method (BEM) and the finite difference method (FDM) [1]. For the time domain, the FDM is frequently used to replace of the derivative item with respect to time. However, these result of FDM is unstable when change the time step size [2].

In 1999, Yang [3] presented the precise time integration algorithm, which not only can obtain the stable and accurate results, but also can check the required number of expansion item by self-adaptive. Up to now, the method combining 
the precise time-domain method with the FEM has been applied to many fields, such as the heat transfer problems [3] and the viscoelastic problems [4]. In addition, the method combining the precise time-domain method with the element free Galerkin method has been applied to the viscoelastic problems [5].

Compared with FEM and FDM, BEM is very robust to solve the heat conduction problems for linear and homogeneous. However, solving the problems of the nonlinear, non-homogeneous and variable coefficients by BEM is difficult, since the fundamental solutions of these problems can hardly obtain, except for some very special cases $[6,7]$. It was a good way that the fundamental solution of the linear problems to solve the problems of the nonlinear, nonhomogeneous, whereas domain integrals was involved in the resulting integral equations.

To overcome this difficulty, the dual reciprocity method (DRM) [8] is used to transform the domain integrals into the boundary integrals. In this method, the transformation is carried out by approximating the source term with a series of basis functions and using their particular solutions. DRM has been extensively solved for the problems of the non-linear and non-homogeneous [9]. The deficiency of this technique is that the particular solutions may be difficult to obtain for some complicated problems. In addition, even for known source terms, the method still requires an approximation of the known function [9].

In 2002, Gao [10] presented a new transformation technique, which is called the radial integration method (RIM). The RIM not only can transform any complicated domain integral to the boundary without using particular solution, but also can remove various singularities appearing in domain integrals [11]. The main feature of the RIM is that it can treat different types of domain integrals appearing in the same integral equation in a unified way, since it does not resort to particular solutions as in the DRM. The method combining the RIM with the BEM is called the radial integration boundary element method (RIBEM) [12].

RIBEM has been widely applied to many fields including dynamic analysis of laminate composite plates [13], nonlinear and nonhomogeneous elastic problems [14], crack analysis in functionally graded materials [15], viscous flow problems $[16]$, and the heat conduction $[12,17]$. However, when solving time-dependent problems via the RIBEM, solutions are sensitive for different time step size due to using finite difference technique to express the derivative term with respect to time.

In this paper, RIBEM and the precise time integration (RIBEM-PTI) algorithm are combined to solve transient heat conduction problems with variable heat conductivity and heat source. By expanding the variables of timedependent in a discrete time interval, RIBEM recursive equation is derived with self-adaptive check technique to improve computing accuracy. Two numerical examples are presented to validate the proposed method with satisfactory results.

\section{Governing equation}

Considering a two-dimensional bounded domain $\Omega$ with a spatially variable heat conductivity and heat source, the governing equation for transient heat 
conduction problems in isotropic media can be expressed as

$$
\frac{\partial}{\partial \mathbf{x}_{i}}\left(k(\mathbf{x}) \frac{\partial T(\mathbf{x}, t)}{\partial \mathbf{x}_{i}}\right)+f(\mathbf{x}, t)=\rho c\left(\frac{\partial T(\mathbf{x}, t)}{\partial t}\right) \quad \mathbf{x} \in \Omega
$$

where $T(\mathbf{x}, t)$ is the temperature at point $\mathbf{x}$ and time $t, k(\mathbf{x})$ denotes the heat conductivity, $f(\mathbf{x}, t)$ is a known heat source, $\rho$ and $c$ represent density and specific heat, respectively. The repeated subscript $i$ denotes the summation through its range which is 2 for two-dimensional problem.

The initial condition is $T(\mathbf{x}, 0)=T_{0}$, where $T_{0}$ is a prescribed function. The boundary conditions are

$$
\begin{gathered}
T(\mathbf{x}, t)=\bar{T}(\mathbf{x}, t) \quad \mathbf{x} \in \Gamma_{1} \\
-k(\mathbf{x}) \frac{\partial T}{\partial n}=\bar{q}(\mathbf{x}, t) \quad \mathbf{x} \in \Gamma_{2}
\end{gathered}
$$

where $\Gamma_{1} \cup \Gamma_{2}=\Gamma, \Gamma$ is boundary of the solving domain $\Omega, \bar{T}$ and $\bar{q}$ are prescribed temperature history and flux on the boundary, respectively.

\section{Recursive governing equation in a discrete time interval}

Within a discrete time interval, time-dependent variables $T, q$ and $f$ can be expanded as

$$
\begin{aligned}
T(\mathbf{x}, t) & =\sum_{m=0} T^{(m)}(\mathbf{x}) s^{m}(t) \\
q(\mathbf{x}, t) & =\sum_{m=0} q^{(m)}(\mathbf{x}) s^{m}(t) \\
f(\mathbf{x}, t) & =\sum_{m=0} f^{(m)}(\mathbf{x}) s^{m}(t)
\end{aligned}
$$

where $s=\left(t-t_{k-1}\right) / \Delta t(k=1,2,3, \ldots), T^{(m)}$ and $q^{(m)}$ are expansion coefficients for the temperature and flux, $f^{(m)}$ is expansion coefficient for the heat source, $t_{k-1}$ and $\Delta t$ denote the beginning time and the size of the time interval, respectively.

The derivative of $T$ with respect to $t$ and $\mathbf{x}$ can be respectively expressed as

$$
\begin{gathered}
\frac{\partial T}{\partial t}=\sum_{m=0} \frac{m+1}{\Delta t} T^{(m+1)} s^{m} \\
\frac{\partial T}{\partial \mathbf{x}_{i}}=\sum_{m=0} \frac{\partial T^{(m)}}{\partial \mathbf{x}_{i}} s^{m}
\end{gathered}
$$

Substituting eqns (6), (7) and (8) into eqn (1), and equating powers of $s^{M}$, then yields

$$
\frac{\partial}{\partial \mathbf{x}_{i}}\left(k(\mathbf{x}) \frac{\partial T^{(M)}}{\partial \mathbf{x}_{i}}\right)+f^{(M)}=\frac{\rho c}{\Delta t}(M+1) T^{(M+1)} \quad \mathbf{x} \in \Omega
$$

eqn (9) is a recursive governing equation with order $M$. 
Assuming that eqns (2) and (3) can be expressed in the form

$$
\begin{array}{ll}
\bar{T}=\sum \bar{T}^{(m)} s^{m} & \text { on } \Gamma_{1} \\
\bar{q}=\sum \bar{q}^{(m)} s^{m} & \text { on } \Gamma_{2}
\end{array}
$$

there are

$$
\begin{array}{ll}
T^{(M)}=\bar{T}^{(M)} & \text { on } \Gamma_{1} \\
q^{(M)}=\bar{q}^{(M)} & \text { on } \Gamma_{2}
\end{array}
$$

eqns (12) and (13) describe boundary conditions of eqn (9) with order $M$.

\section{Implementation of RIBEM}

\subsection{Boundary-domain integral equation}

To derive the boundary integral equation, a weight function $G$ is introduced to eqn (9) and the following domain integral can be written

$$
\int_{\Omega} G \frac{\partial}{\partial \mathbf{x}_{i}}\left(k(\mathbf{x}) \frac{\partial T^{(M)}}{\partial \mathbf{x}_{i}}\right) d \Omega+\int_{\Omega} G f^{(M)} d \Omega=(M+1) \frac{\rho c}{\Delta t} \int_{\Omega} G T^{(M+1)} d \Omega
$$

Using Gauss' divergence theorem, the first domain integral can be manipulated as

$$
\begin{aligned}
& \int_{\Omega} G(\mathbf{x}, \mathbf{y}) \frac{\partial}{\partial \mathbf{x}_{i}}\left(k(\mathbf{x}) \frac{\partial T^{(M)}}{\partial \mathbf{x}_{i}}\right) d \Omega=\int_{\Gamma} G(\mathbf{x}, \mathbf{y}) k(\mathbf{x}) \frac{\partial T^{(M)}}{\partial \mathbf{x}_{i}} n_{i} d \Gamma \\
& -\int_{\Gamma} k(\mathbf{x}) T^{(M)} \frac{\partial G(\mathbf{x}, \mathbf{y})}{\partial \mathbf{x}_{i}} n_{i} d \Gamma+\int_{\Omega} T^{(M)} \frac{\partial G(\mathbf{x}, \mathbf{y})}{\partial \mathbf{x}_{i}} \frac{\partial k(\mathbf{x})}{\partial \mathbf{x}_{i}} d \Omega \\
& +\int_{\Omega} k(\mathbf{x}) T^{(M)} \frac{\partial}{\partial \mathbf{x}_{i}}\left(\frac{\partial G(\mathbf{x}, \mathbf{y})}{\partial \mathbf{x}_{i}}\right) d \Omega
\end{aligned}
$$

If the weight function $G$ is Green's function $G(\mathbf{x}, \mathbf{y})$ which satisfies the following equation:

$$
\nabla^{2} G+\delta(\mathbf{x}-\mathbf{y})=0
$$

where $\delta(\mathbf{x}-\mathbf{y})$ is the Dirac delta function, according to literature [18], the Green's function $G(\mathbf{x}, \mathbf{y})$ for eqn (16) can be expressed as $G(\mathbf{x}, \mathbf{y})=-(\ln r) / 2 \pi$, where $r$ is the distance between the source point $\mathbf{y}$ and the field point $\mathbf{x}$.

Based on the integration property of the Dirac delta function, the last domain integral of eqn (15) can be written as

$$
\int_{\Omega} k(\mathbf{x}) T^{(M)} \frac{\partial}{\partial \mathbf{x}_{i}}\left(\frac{\partial G(\mathbf{x}, \mathbf{y})}{\partial \mathbf{x}_{i}}\right) d \Omega=-k(\mathbf{y}) T^{(M)}(\mathbf{y})
$$

Substituting eqn (17) into eqn (15) and the result into eqn (14), it follows that 


$$
\begin{aligned}
\tilde{T}^{(M)}(\mathbf{y})= & -\int_{\Gamma} G(\mathbf{x}, \mathbf{y}) q^{(M)}(\mathbf{x}) d \Gamma-\int_{\Gamma} \frac{\partial G(\mathbf{x}, \mathbf{y})}{\partial n} \tilde{T}^{(M)}(\mathbf{x}) d \Gamma \\
& +\int_{\Omega} G(\mathbf{x}, \mathbf{y}) f^{(M)} d \Omega+\int_{\Omega} V(\mathbf{x}, \mathbf{y}) \tilde{T}^{(M)}(\mathbf{x}) d \Omega \\
& -(M+1) \frac{\rho c}{\Delta t} \int_{\Omega} \frac{G(\mathbf{x}, \mathbf{y})}{k(\mathbf{x})} \tilde{T}^{(M+1)}(\mathbf{x}) d \Omega
\end{aligned}
$$

where $q^{(M)}(\mathbf{x})$ is the heat flux, $\tilde{T}^{(M)}(\mathbf{x})$ and $\tilde{k}(\mathbf{x})$ are the normalized temperature and thermal conductivity, respectively, e.g.

$$
\begin{aligned}
& q^{(M)}(\mathbf{x})=-k(\mathbf{x}) \partial T^{(M)} / \partial n ; \quad \tilde{T}^{(M)}(\mathbf{x})=k(\mathbf{x}) T^{(M)}(\mathbf{x}) ; \\
& \tilde{k}(\mathbf{x})=\ln k(\mathbf{x}) ; V(\mathbf{x}, \mathbf{y})=\left(\partial G(\mathbf{x}, \mathbf{y}) / \partial \mathbf{x}_{i}\right) \cdot\left(\partial \tilde{k}(\mathbf{x}) / \partial \mathbf{x}_{i}\right)
\end{aligned}
$$

A general integral equation is presented by letting $\mathbf{y} \rightarrow \Gamma$ as follows:

$$
\begin{aligned}
c(\mathbf{y}) \tilde{T}^{(M)}(\mathbf{y})=-\int_{\Gamma} G(\mathbf{x}, \mathbf{y}) q^{(M)}(\mathbf{x}) \mathrm{d} \Gamma-\int_{\Gamma} \frac{\partial G(\mathbf{x}, \mathbf{y})}{\partial n} \tilde{T}^{(M)}(\mathbf{x}) \mathrm{d} \Gamma \\
+\int_{\Omega} G(\mathbf{x}, \mathbf{y}) f^{(M)} \mathrm{d} \Omega+\int_{\Omega} V(\mathbf{x}, \mathbf{y}) \tilde{T}^{(M)}(\mathbf{x}) \mathrm{d} \Omega \\
-(M+1) \frac{\rho c}{\Delta t} \int_{\Omega} \frac{G(\mathbf{x}, \mathbf{y})}{k(\mathbf{x})} \tilde{T}^{(M+1)}(\mathbf{x}) d \Omega
\end{aligned}
$$

where $c(\mathbf{y})=\varphi(\mathbf{y}) / 2 \pi, \varphi(\mathbf{y})$ is the interior angle at a point $\mathbf{y}$ of the boundary $\Gamma$. Particularly, $c(\mathbf{y})=0.5$ if $\mathbf{y}$ is a smooth point on the boundary.

\subsection{Transformation of domain integrals to the boundary by RIM}

In general, the heat source $f(\mathbf{x}, t)$ is a known function. Therefore, $f^{(M)}$ can be obtained by expanding coefficients of $f(\mathbf{x}, t)$ in a time interval. In these circumstances, RIM [10] can be directly used to transform the first domain integral in eqn (19) to the boundary as follows:

$$
\int_{\Omega} G(\mathbf{x}, \mathbf{y}) f^{(M)} d \Omega=\int_{\Gamma} \frac{1}{r(\mathbf{z}, \mathbf{y})} \frac{\partial r}{\partial n} F^{(M)}(\mathbf{z}, \mathbf{y}) d \Gamma
$$

where

$$
F^{(M)}(\mathbf{z}, \mathbf{y})=\int_{0}^{r(\mathbf{z}, \mathbf{y})} G(\mathbf{x}, \mathbf{y}) f^{(M)} r d r
$$

For the last two domain integrals in eqn (19), since the normalized temperature $\tilde{T}^{(M)}$ and $\tilde{T}^{(M+1)}$ are involved, which are unknown, the RIM formulations cannot be directly used. To solve this problem, $\tilde{T}^{(M)}$ and $\tilde{T}^{(M+1)}$ are approximated by the combination of the radial basis functions (RBFs) and the polynomials in terms of global coordinates $[12,14]$. Thus, $\tilde{T}^{(M)}$ and $\tilde{T}^{(M+1)}$ are expressed as follows [12]: 


$$
\begin{aligned}
& \tilde{T}^{(M)}=\sum_{k=1}^{N} \alpha_{k} \phi_{k}(R)+a_{1} \mathbf{x}_{1}+a_{2} \mathbf{x}_{2}+a_{3} \\
& \tilde{T}^{(M+1)}=\sum_{k=1}^{N} \beta_{k} \phi_{k}(R)+b_{1} \mathbf{x}_{1}+b_{2} \mathbf{x}_{2}+b_{3}
\end{aligned}
$$

The following equilibrium conditions have to be satisfied [19]

$$
\begin{aligned}
& \sum_{k=1}^{N} \alpha_{k}=\sum_{k=1}^{N} \alpha_{k} \mathbf{x}_{1}^{k}=\sum_{k=1}^{N} \alpha_{k} \mathbf{x}_{2}^{k}=0 \\
& \sum_{k=1}^{N} \beta_{k}=\sum_{k=1}^{N} \beta_{k} \mathbf{x}_{1}^{k}=\sum_{k=1}^{N} \beta_{k} \mathbf{x}_{2}^{k}=0
\end{aligned}
$$

where $N=N_{b}+N_{I}$ and $N_{b}, N_{I}$ are the number of boundary and interior nodes, respectively, $R=\left\|\mathbf{x}-\mathbf{x}^{k}\right\|$ is the distance from application point $\mathbf{x}^{k}$ to the field point $\mathbf{x}, \mathbf{x}_{i}^{k}$ is the coordinates of each of the $k$-th field point, $\phi(R)$ is the RBF. In this paper, the compactly supported fourth-order spline RBF is adopted, e.g.

$$
\phi_{k}(R)=\left\{\begin{array}{cc}
1-6\left(R / d_{k}\right)^{2}+8\left(R / d_{k}\right)^{3}-3\left(R / d_{k}\right)^{4} & 0 \leq R<d_{k} \\
0 & d_{k} \leq R
\end{array}\right.
$$

in which $d_{k}$ is radius of the supported region at the $k$-th point.

The coefficients $\alpha_{k}$ for $k=1,2, \cdots, N, a_{1}, a_{2}$ and $a_{3}$ in eqn (22) can be determined by collocating the application point $\mathbf{x}$ in eqn (22) at all nodes. A set of algebraic equations can be written in the matrix form as $\tilde{\mathbf{T}}_{\alpha}^{M}=\boldsymbol{\phi} \alpha$, where $\boldsymbol{\alpha}=\left\{\alpha_{1}, \alpha_{2}, \cdots, \alpha_{N}, a_{1}, a_{2}, a_{3}\right\}^{\mathrm{T}}, \tilde{\mathbf{T}}_{\alpha}^{(M)}=\left\{\tilde{T}_{1}^{(M)}, \tilde{T}_{2}^{(M)}, \cdots, \tilde{T}_{N}^{(M)}, 0,0,0\right\}^{\mathrm{T}}$. If no two nodes share the same coordinates, the matrix $\phi$ is invertible and thereby

$$
\boldsymbol{\alpha}=\boldsymbol{\phi}^{-1} \tilde{\mathbf{T}}_{\alpha}^{(M)}
$$

For $\tilde{T}^{(M+1)}$ in eqn (23), a similar expression is adopted, e.g.

$$
\begin{aligned}
& \tilde{\mathbf{T}}_{\beta}^{(M+1)}=\boldsymbol{\phi} \boldsymbol{\beta} \\
& \boldsymbol{\beta}=\boldsymbol{\phi}^{-1} \tilde{\mathbf{T}}_{\beta}^{(M+1)}
\end{aligned}
$$

where $\boldsymbol{\beta}=\left\{\beta_{1}, \beta_{2}, \cdots \beta_{N}, b_{1}, b_{2}, b_{3}\right\}^{\mathrm{T}}, \tilde{\mathbf{T}}_{\beta}^{(M+1)}=\left\{\tilde{T}_{1}^{(M+1)}, \tilde{T}_{2}^{(M+1)}, \cdots, \tilde{T}_{N}^{(M+1)}, 0,0,0\right\}^{\mathrm{T}}$.

Substituting eqn (22) into the second domain integral in eqn (19) and transforming it to the boundary applying the RIM, the following results can be obtained:

$$
\begin{array}{r}
\int_{\Omega} V(\mathbf{x}, \mathbf{y}) \tilde{T}^{(M)}(\mathbf{x}) d \Omega=\sum_{k=1}^{N} \alpha_{k} \int_{\Gamma} \frac{1}{r} \frac{\partial r}{\partial n} \bar{F}_{k} d \Gamma+\sum_{i=1}^{2} a_{i} \int_{\Gamma} \frac{r_{, i}}{r} \frac{\partial r}{\partial n} \bar{F}_{V 1} d \Gamma+ \\
\left(\sum_{i=1}^{2} a_{i} \mathbf{y}_{i}+a_{3}\right) \int_{\Gamma} \frac{1}{r} \frac{\partial r}{\partial n} \bar{F}_{V 2} d \Gamma=\mathbf{V}_{\mathbf{y}} \tilde{\mathbf{T}}^{(M)}
\end{array}
$$


where

$$
\left\{\begin{array}{l}
\bar{F}_{k}=\int_{0}^{r(\mathbf{z}, \mathbf{y})} V(\mathbf{x}, \mathbf{y}) \phi_{k}(R) r d r \\
\bar{F}_{V 1}=\int_{0}^{r(\mathbf{z}, \mathbf{y})} V(\mathbf{x}, \mathbf{y}) r^{2} d r \\
\bar{F}_{V 2}=\int_{0}^{r(\mathbf{z}, \mathbf{y})} V(\mathbf{x}, \mathbf{y}) r d r
\end{array}\right.
$$

$\tilde{\mathbf{T}}^{(M)}=\left\{\tilde{T}_{1}^{(M)}, \tilde{T}_{2}^{(M)}, \cdots, \tilde{T}_{N}^{(M)}\right\}^{\mathrm{T}}, \mathbf{V}_{\mathbf{y}}$ is a $N$ dimensional row vector.

Substituting eqn (23) into the last domain integral in eqn (19) and transforming it to the boundary applying the RIM, the similar expression can be presented as

$$
\begin{aligned}
\int_{\Omega} \frac{G(\mathbf{x}, \mathbf{y})}{k(\mathbf{x})} \tilde{T}^{(M+1)} d \Omega & =\sum_{k=1}^{N} \beta_{k} \int_{\Gamma} \frac{1}{r} \frac{\partial r}{\partial n} \overline{\bar{F}}_{k} d \Gamma+\sum_{i=1}^{2} b_{i} \int_{\Gamma} \frac{r_{i}}{r} \frac{\partial r}{\partial n} \overline{\bar{F}}_{G 1} d \Gamma \\
& +\left(\sum_{i=1}^{2} b_{i} \mathbf{y}_{i}+b_{3}\right) \int_{\Gamma} \frac{1}{r} \frac{\partial r}{\partial n} \overline{\bar{F}}_{G 2} d \Gamma=\mathbf{C}_{\mathbf{y}} \tilde{\mathbf{T}}^{(M+1)}
\end{aligned}
$$

where

$$
\left\{\begin{array}{c}
\overline{\bar{F}}_{k}=\int_{0}^{r(\mathbf{z}, \mathbf{y})} \frac{G(\mathbf{x}, \mathbf{y})}{k(\mathbf{x})} \phi_{k}(R) r d r \\
\overline{\bar{F}}_{G 1}=\int_{0}^{r(\mathbf{z}, \mathbf{y})} \frac{G(\mathbf{x}, \mathbf{y})}{k(\mathbf{x})} r^{2} d r \\
\overline{\bar{F}}_{G 2}=\int_{0}^{r(\mathbf{z}, \mathbf{y})} \frac{G(\mathbf{x}, \mathbf{y})}{k(\mathbf{x})} r d r
\end{array}\right.
$$

Also, $\tilde{\mathbf{T}}^{(M+1)}=\left\{\tilde{T}_{1}^{(M+1)}, \tilde{T}_{2}^{(M+1)}, \cdots, \tilde{T}_{N}^{(M+1)}\right\}^{\mathrm{T}}, \mathbf{C}_{\mathbf{y}}$ is a $N$ dimensional row vector. The radial integrals in eqns (21) (31) and (33) can be evaluated by numerical method [10].

Substituting eqns (20), (30) and (32) into eqn (19), a pure boundary integral equation is obtained as follows:

$$
\begin{aligned}
c(\mathbf{y}) \tilde{T}^{(M)}(\mathbf{y})= & -\int_{\Gamma} G(\mathbf{x}, \mathbf{y}) q^{(M)}(\mathbf{x}) d \Gamma-\int_{\Gamma} \frac{\partial G(\mathbf{x}, \mathbf{y})}{\partial n} \tilde{T}^{(M)}(\mathbf{x}) d \Gamma \\
& +\int_{\Gamma} \frac{1}{r} \frac{\partial r}{\partial n} F^{(M)} d \Gamma+\mathbf{V}_{\mathbf{y}} \tilde{\mathbf{T}}^{(M)}-(M+1) \frac{\rho c}{\Delta t} \mathbf{C}_{\mathbf{y}} \tilde{\mathbf{T}}^{(M+1)}
\end{aligned}
$$

\subsection{Algebraic system of equations}

Assuming that the boundary $\Gamma$ is discretized into $N_{b}$ linear elements and the region is distributed $N_{I}$ internal nodes, the total number of nodes is $N=N_{b}+N_{I}$. Eqn (34) can be conveniently expressed in the following matrix form [12]: 


$$
\begin{aligned}
& \mathbf{C}_{\alpha} \tilde{\mathbf{T}}_{b}^{(M)}=\mathbf{G}_{b} \mathbf{Q}_{b}^{(M)}-\hat{\mathbf{H}}_{b} \tilde{\mathbf{T}}_{b}^{(M)}+\mathbf{f}_{b}^{(M)}+\mathbf{V}_{b} \tilde{\mathbf{T}}^{(M)}-(M+1) \frac{\rho c}{\Delta t} \mathbf{C}_{b} \tilde{\mathbf{T}}^{(M+1)} \text { on } \Gamma \\
& \tilde{\mathbf{T}}_{I}^{(M)}=\mathbf{G}_{I} \mathbf{Q}_{b}^{(M)}-\hat{\mathbf{H}}_{I} \tilde{\mathbf{T}}_{b}^{(M)}+\mathbf{f}_{I}^{(M)}+\mathbf{V}_{I} \tilde{\mathbf{T}}^{(M)}-(M+1) \frac{\rho c}{\Delta t} \mathbf{C}_{I} \tilde{\mathbf{T}}^{(M+1)} \text { in } \Omega
\end{aligned}
$$

where $\quad \mathbf{C}_{\alpha}=\operatorname{diag}\left\{c\left(\mathbf{y}_{b 1}\right), c\left(\mathbf{y}_{b 2}\right), \cdots, c\left(\mathbf{y}_{N_{b}}\right)\right\}, \quad \tilde{\mathbf{T}}_{b}^{(M)}=\left\{\tilde{T}_{b 1}^{(M)}, \tilde{T}_{b 2}^{(M)}, \cdots, \tilde{T}_{N_{b}}^{(M)}\right\}^{\mathrm{T}}$, $\mathbf{Q}_{b}^{(M)}=\left\{\partial T_{b 1}^{(M)} / \partial n, \partial T_{b 2}^{(M)} / \partial n, \cdots, \partial T_{N_{b}}^{(M)} / \partial n\right\}^{\mathrm{T}}$, $\mathbf{f}_{b}^{(M)}=\left\{f_{1}^{(M)}, f_{2}^{(M)}, \cdots, f_{N_{b}}^{(M)}\right\}^{\mathrm{T}} \tilde{\mathbf{T}}_{I}^{(M)}=\left\{\tilde{T}_{I 1}^{(M)}, \tilde{T}_{I 2}^{(M)}, \cdots, \tilde{T}_{N_{I}}^{(M)}\right\}^{\mathrm{T}}$, $\mathbf{f}_{I}^{(M)}=\left\{f_{1}^{(M)}, f_{2}^{(M)}, \cdots, f_{N_{I}}^{(M)}\right\}^{\mathrm{T}}$, the matrices $\mathbf{G}_{b}, \hat{\mathbf{H}}_{b}, \mathbf{G}_{I}$ and $\hat{\mathbf{H}}_{I}$ correspond to the coefficients of boundary integrals, $\mathbf{f}_{b}^{M}, \mathbf{V}_{b}, \mathbf{C}_{b}, \mathbf{f}_{I}^{M}, \mathbf{V}_{I}$ and $\mathbf{C}_{I}$ refer to the coefficients of domain integrals term.

After the application of boundary conditions, eqns (35) and (36) can be recast as

$$
\begin{aligned}
& \mathbf{A}_{b} \mathbf{X}_{b}^{(M)}=\mathbf{Y}_{b}^{(M)}+\mathbf{V}_{b} \tilde{\mathbf{T}}^{(M)}-(M+1) \frac{\rho c}{\Delta t} \mathbf{C}_{b} \tilde{\mathbf{T}}^{(M+1)} \quad \text { on } \Gamma \\
& \tilde{\mathbf{T}}_{I}^{(M)}=\mathbf{A}_{I} \mathbf{X}_{b}^{(M)}+\mathbf{Y}_{I}^{(M)}+\mathbf{V}_{I} \tilde{\mathbf{T}}^{(M)}-(M+1) \frac{\rho c}{\Delta t} \mathbf{C}_{I} \tilde{\mathbf{T}}^{(M+1)} \text { in } \Omega
\end{aligned}
$$

where the dimensions of the coefficient matrix $\mathbf{A}_{b}$ and $\mathbf{A}_{I}$ are $N_{b} \times N_{b}$ and $N_{I} \times N_{b}$, respectively; $\mathbf{V}_{b}$ and $\mathbf{C}_{b}$ are $N_{b} \times N ; \mathbf{V}_{I}$ and $\mathbf{C}_{I}$ are $N_{I} \times N$. Vector $\mathbf{X}_{b}^{M}$ with the dimension of $N_{b}$ consists of unknown boundary temperatures or unknown fluxes. $\mathbf{Y}_{b}^{(M)}$ and $\mathbf{Y}_{I}^{(M)}$ are the contribution of known boundary variables. It is noted that the integration results $\mathbf{f}_{b}^{(M)}$ and $\mathbf{f}_{I}^{(M)}$ have been assembled into the known vectors $\mathbf{Y}_{b}^{(M)}$ and $\mathbf{Y}_{I}^{(M)}$ in eqns (37) and (38).

\subsection{Self-adaptive check}

In the first time interval, $\tilde{T}^{0}$ or $q^{0}$ are provided by eqns (12) and (13), in other time intervals, initial conditions will be given by

$$
\begin{aligned}
& \tilde{T}_{s=1}=\sum_{m=0} \tilde{T}^{(m)} \text { (at the last time interval) } \\
& q_{s=1}=\sum_{m=0} q^{(m)} \text { (at the last time interval) }
\end{aligned}
$$

By using proper initial conditions and eqns (37) and (38), the transient behavior of temperature can be traced step by step.

A self-adaptive scheme is carried out at each of time intervals with a convergence criteria 


$$
\left\|\mathbf{X}^{(m)}\right\|_{2} /\left\|\sum_{j=0}^{m-1} \mathbf{X}^{(j)}\right\|_{2} \leq \varepsilon
$$

where the vector $\mathbf{X}$ is obtained by eqns (37) and (38), $\varepsilon$ is an error bound, $m$ is calculated times in present time interval, $\|\bullet\|_{2}$ represents a vector 2-norm.

In this paper, $\varepsilon=10^{-3}$ is adopted. If eqn (41) is satisfied continually 3 times, computing will stop in the time interval considered, and step into next one. The order of expansion can be automatically adapted in present time step.

\section{Numerical examples}

To check the convergence of the proposed method, the root mean square (RMS) error is given by

$$
R M S=\sqrt{\sum_{i=1}^{N}\left(T_{\text {numerical }, i}-T_{\text {exact }, i}\right)^{2} / \sum_{i=1}^{N} T_{\text {exact }, i}^{2}}
$$

where $T_{\text {numerical }, i}$ and $T_{\text {exact }, i}$ are the numerical solution and the exact solution of the $i$-th node, respectively. For comparison, examples are also computed using the RIBEM, which use the finite difference technique to simulate the derivative of temperature with respect to time (it will be abbreviated to RIBEM-FD). RIBEM-FD was described in detail in the literature [12] where the Euler factor equals one. Finally, a concave geometry is discussed.

Example 1: Consider a square plate $\Omega=[0, \pi]^{2}$ with $k=\rho=c=1$. The plate is discretized into 32 equally space linear boundary elements and distributed uniformly 49 internal nodes. The heat source is $f(\mathbf{x}, t)=e^{-t} \sin \mathbf{x}_{1} \sin \mathbf{x}_{2}$, the initial condition is $T_{0}=\sin \mathbf{x}_{1} \sin \mathbf{x}_{2}$ and the boundary condition is $T(\Gamma)=0$. The exact solution of the temperature field can be expressed as $T(\mathbf{x}, t)=e^{-t} \sin \mathbf{x}_{1} \sin \mathbf{x}_{2}$.

Fig. 1 shows the RMS errors of the temperature for the RIBEM-PTI and the RIBEM-FD. The results indicate that the present approach can effectively solve the problem with the heat source and can obtain more stable and accurate results than the RIBEM-FD.

Example 2: Consider a square plate $\Omega=[1,2]^{2}$ with $k(\mathbf{x})=\mathbf{x}_{1}+\mathbf{x}_{2}, \rho=c=1$. The plate is discretized into 20 equally space linear boundary elements and distributed uniformly 16 internal nodes. The heat source is $f(\mathbf{x}, t)=-6\left(\mathbf{x}_{1}+\mathbf{x}_{2}\right)+2 t$ and the initial condition is $T_{0}=\left(\mathbf{x}_{1}\right)^{2}+\left(\mathbf{x}_{2}\right)^{2}$. The boundary conditions are $T\left(\mathbf{x}_{1}, 1, t\right)=\left(\mathbf{x}_{1}\right)^{2}+1+t^{2}, \quad T\left(2, \mathbf{x}_{2}, t\right)=4+\left(\mathbf{x}_{2}\right)^{2}+t^{2}$, $T\left(\mathbf{x}_{1}, 2, t\right)=\left(\mathbf{x}_{1}\right)^{2}+4+t^{2}$ and $T\left(1, \mathbf{x}_{2}, t\right)=1+\left(\mathbf{x}_{2}\right)^{2}+t^{2}$. The exact solution of the temperature field can be expressed as $T(\mathbf{x}, t)=\left(\mathbf{x}_{1}\right)^{2}+\left(\mathbf{x}_{2}\right)^{2}+t^{2}$.

Fig. 2 shows the RMS errors of the temperature for the RIBEM-PTI and the RIBEM-FD. The results indicate that the present approach can effectively solve 
the problem with the varying heat conductivity and can obtain more stable and accurate results than the RIBEM-FD, even though more refined time steps are used in the RIBEM-FD.

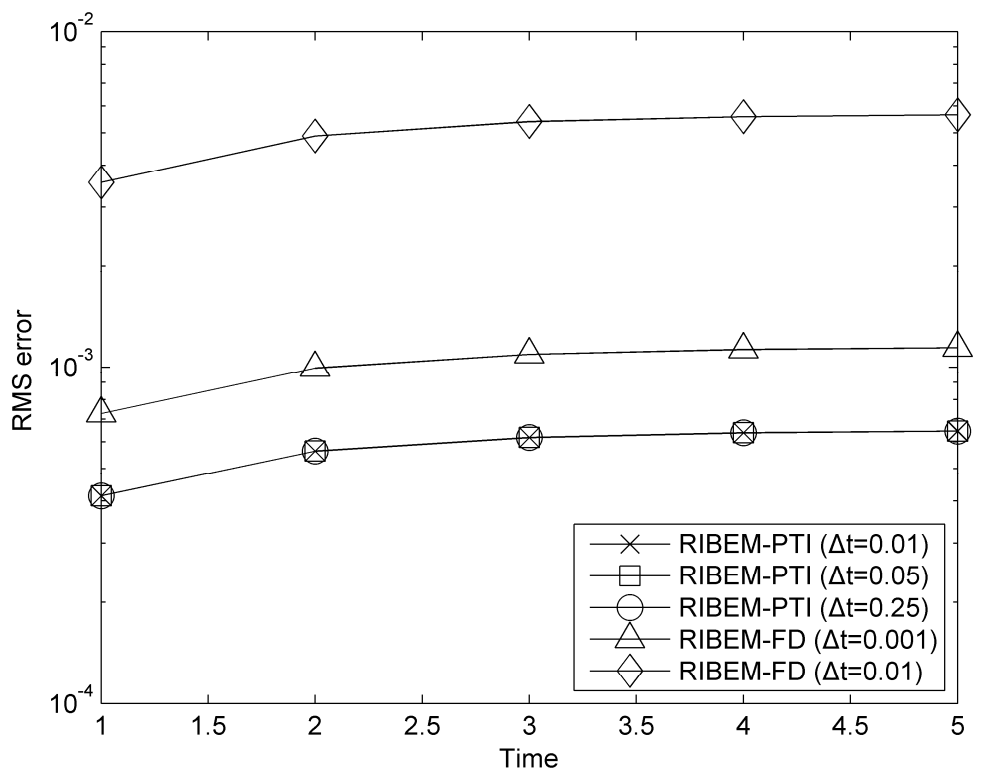

Figure 1: $\quad$ RMS error of temperature for Example 1.

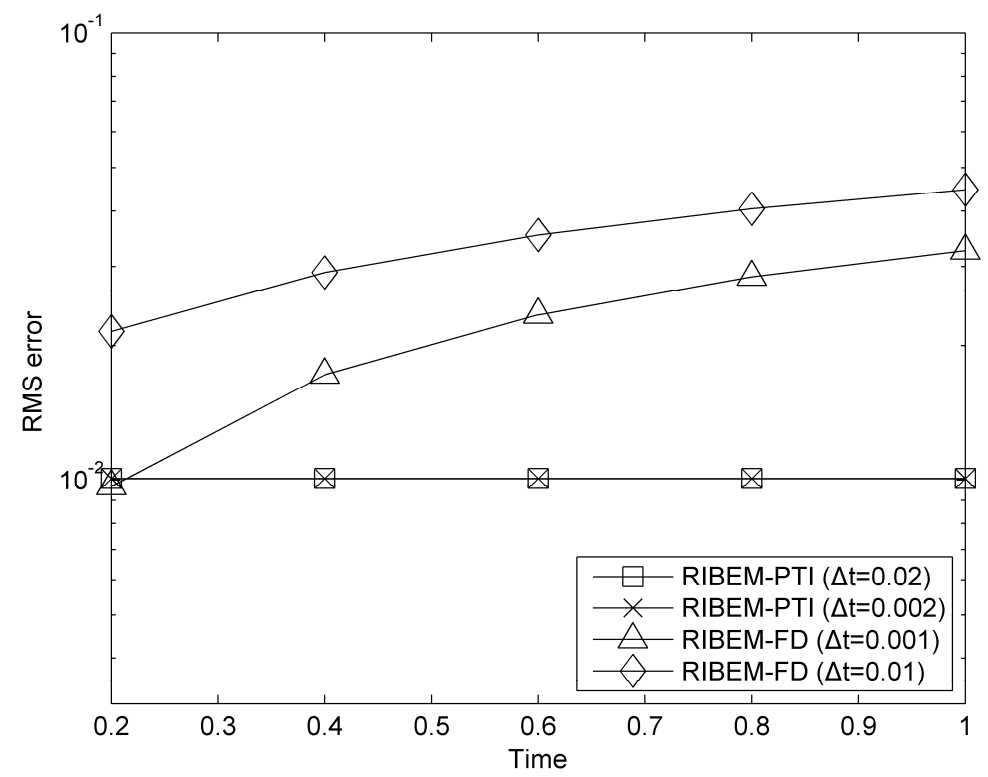

Figure 2: $\quad$ RMS error of temperature for Example 2. 


\section{Conclusions}

In this paper, the precise time integration algorithm is introduced to the radial integration BEM for solving the transient heat conduction problems with varying heat conductivity. For the RIBEM-FD, the sensitive results are caused by the finite difference method to solve the derivative of temperature with respect to time. The RIBEM-PTI can perfectly be used to solve the problem by expanding variables in the time interval. Numerical results show that the RIBEM-PTI can obtain satisfactory results and inherit merits of the RIBEM. Moreover, the RIBEM-PTI can obtain the relatively stable and accurate results for a big time step, the RIBEM-FD can obtain accurate results only in the case of a small time step.

\section{Acknowledgement}

The work described in this paper was supported by the Specialized Research Fund for the Doctoral Program of Higher Education (No. 20120041110014).

\section{References}

[1] Minkowycz, W.J., Sparrow, E.M. \& Murthy, J.Y., Handbook of Numerical Heat Transfer, Wiley, 2006.

[2] Özisik, M.N., Finite Difference methods in Heat Transfer. CRC Press, 1994.

[3] Yang, H.T., A precise algorithm in the time domain to solve the problem of heat transfer. Numerical Heat Transfer B, 35(2), pp. 243-249, 1999.

[4] Ren, Y. \& Yang, H.T., Equivalent analysis of orthogonal viscoelastic jointed rock via an adaptive algorithm in time domain. Finite Elements in Analysis Design, 46(10), pp. 875-888, 2010.

[5] Yang, H.T. \& Liu, Y., A combined approach of EFGM and precise algorithm in time domain solving viscoelasticity problem. International journal of solids and structures, 40(3), pp. 701-714, 2003.

[6] Ang, W.T., Kusuma, J. \& Clements, D.L., A boundary element method for a second order elliptic partial differential equation with variable coefficients. Engineering Analysis with Boundary Elements, 18(4), pp. 311-316, 1996.

[7] Clements D.L., Fundamental solutions for second order linear elliptic partial differential equations. Computational Mechanics, 22(1), pp. 26-31, 1998.

[8] Nardini D. \& Brebbia C.A., A new approach for free vibration analysis using boundary elements. In: Brebbia CA, editor. Boundary Element Methods in Engineering. Berlin: Springer, pp. 312-326, 1982.

[9] Partridge, P.W., Brebbia, C.A. \& Wrobel, L.C., The dual reciprocity boundary element method, Southampton: Computational Mechanics Publication, 1992. 
[10] Gao, X.W., The radial integration method for evaluation of domain integrals with boundary-only discretization. Engineering Analysis with Boundary Elements, 26(10), pp. 905-916, 2002.

[11] Gao, X.W., Evaluation of regular and singular domain integral with boundary-only discretization-theory and Fortran code. Journal of Computational and Applied Mathematics, 175(2), pp. 265-290, 2005.

[12] Yang, K. \& Gao, X.W., Radial integration BEM for transient heat conduction problems. Engineering Analysis with Boundary Elements, 34(6), 557-563, 2010.

[13] Albuquerque, E.L., Sollero, P. \& Portilho de Paiva, W., The BEM and the RIM in the dynamic analysis of symmetric laminate composite plates. Brazilian Society of Mechanical Sciences and Engineering, 41-50, 2007.

[14] Gao, X.W., Zhang, C. \& L. Guo, Boundary-only element solutions of 2D and 3D nonlinear and nonhomogeneous elastic problems. Engineering Analysis with Boundary Elements, 31(12), pp. 974-982, 2007.

[15] Zhang, C., Cui, M., Wang, J., Gao, X.W., Sladek, J. and Sladek, V., 3D crack analysis in functionally graded materials. Engineering Fracture Mechanics, 78(3), pp. 585-604, 2011.

[16] Peng, H.F., Cui, M. \& Gao, X.W., A boundary element method without internal cells for solving viscous flow problems. Engineering Analysis with Boundary Elements, 37, pp. 293-300, 2013.

[17] AL-Jawary, M.A. \& Wrobel, L.C., Radial integration boundary integral and integro-differential equation methods for two-dimensional heat conduction problems with variable coefficients. Engineering Analysis with Boundary Elements, 36(5), pp. 685-695, 2012.

[18] Brebbia, C.A. \& Dominguez, J., Boundary elements an introductory course, 2nd ed. Boston: Computational Mechanics Publications, 1992.

[19] Karur, S.R., Ramachandran, P.A., Augmented thin plate spline approximation in DRM. Boundary Elements Communications, 6, pp. 55$58,1995$. 